\title{
Transmission Diffractive Microscopy Without Lenses at Visible, X-ray and Electron Wavelengths
}

\author{
J.M. Rodenburg, ${ }^{*}$ A.M. Maiden, ${ }^{*}$ D. Batey, ${ }^{*}$ F. Sweeney, ${ }^{*}$ T.B. Edo, ${ }^{*}$ A.C. Hurst, ${ }^{*}$ F. Hüe, ${ }^{* *}$ \\ P.A. Midgley, ${ }^{* *}$ P. Wang***, A.I. Kirkland***, and M.J. Humphry.**** \\ * Department of Electronic and Electrical Engineering, Sir Frederick Mappin Building, Mappin \\ Street, Sheffield, S1 3JD, UK († deceased) \\ ** Department of Materials Science and Metallurgy, University of Cambridge, Pembroke Street, \\ Cambridge, CB2 3QZ, UK \\ *** Department of Materials, University of Oxford, Parks Road, Oxford, OX1 3PH, UK \\ **** Phase Focus Ltd, Kroto Innovation Centre, University of Sheffield, Sheffield, S3 7HQ, UK
}

The biggest challenge faced by all forms of atomic-scale transmission microscopy is the manufacture of good quality lenses that can re-interfere wave components with picometre-scale accuracy. Even with aberration-correction, the resolution of electron optics is still very poor relative to the wavelength of a high-energy electron. In the case of Fresnel zone plate X-ray imaging, resolution is limited to the accuracy of manufacture of the outer ring. Coherent diffractive imaging (CDI) disposes with the lens entirely and substitutes the imaging process by a computation that recovers the phase of the intensity of the scattered (diffracted) waves. The original method of achieving this was to use a-priori information relating to the size of the object of interest in order to constrain the number of solutions that were consistent with both recorded diffracted intensity and the known object size constraint [1,2]. A much more robust and useful solution method is via ptychography, in which a number of diffraction patterns are recorded as a patch of illumination is scanned to a number of positions over the object. This allows a wide (indefinite) field of view to be obtained at a resolution determined by the effective numerical aperture of the detector (that is, the sine of the angle subtended at the sample plane by opposite edges of the detector).

Although there were proofs-of-principle for visible light and electron ptychography during the 1990 s, only with the advent of a practical and extremely fast phase-retrieval algorithm was it possible to demonstrate the approach convincingly with light [3] and then with X-rays [4]. The method has now become almost a standard technique at some synchrotron light sources, and has even been extended to 3D X-ray tomography [5].

It turns out that even at visible light wavelengths (where of course very good quality lenses are available), there are some uniquely valuable properties of ptychography. For example, it can recover the phase of the waves that have traversed the object with exquisite sensitivity. Because the complex exit wave (or at least, a quantity closely related to the exit wave) is recovered, it is possible to focus on different layers of the object retrospectively. Finally, very large working distances are possible, achieving good resolution through thick containers, such as Petri dishes, and in liquids.

It is in electron microscopy where the greatest gains in performance over existing imaging methods are likely to occur. We have demonstrated a proof-of-principle of wide-field-of-view electron ptychography, but at the time of writing, have only achieved a resolution of about $1 \mathrm{~nm}$, although the phase sensitivity of the image is very good [6]. We will report latest developments in both electron and light ptychography. 


\section{References}

[1] J.Miao, et al., Nature, 400 (1999) 342

[2] J.M.Zuo, et al., Science 300 (2003) 1419

[3] J.M.Rodenburg et al., Ultramicroscopy, 107 (2007) 227

[4] J.M.Rodenburg et al., Phys. Rev. Lett. 98 (2007) 034801

[5] M.Dierolf, et al., Nature, 467 (2010) 436

[6] F.Hue, et al., Phys. Rev. B, 82 (2010) 121415(R)

[7] This work was funded by EPSRC Basic Technology Grant. No EP/E034055/1, Pi-Phi project, and Phase Focus Ltd.

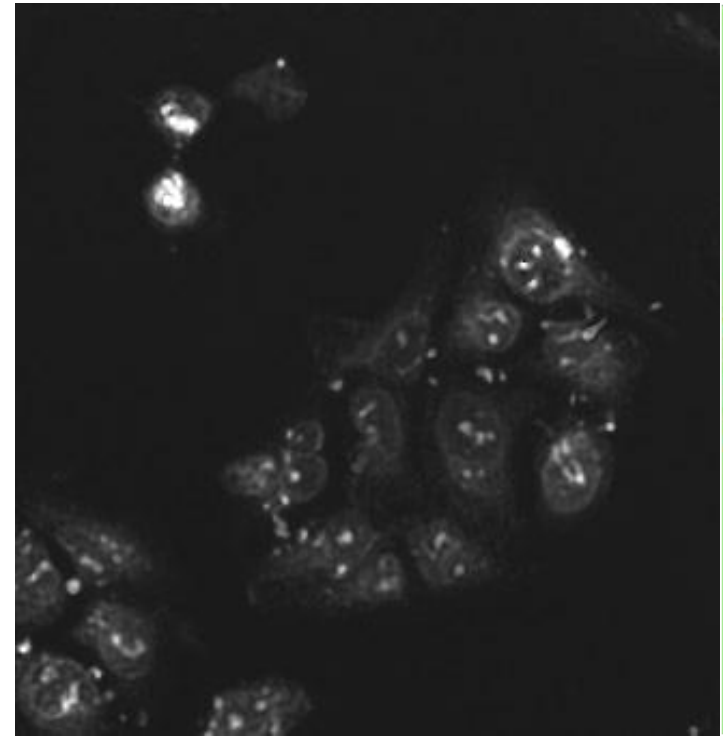

Top left (field of view $350 \mathrm{m \mu}$ ): Visible light ptychograph of biological cells. We display phase of the image: note very high contrast on a flat black background. Unlike Zernike contrast, there are no artifacts in the image; what we see here is a direct plot of the optical thickness of the specimen.

Top right (field of view 14mm): a phase ptychograph of a toric contact lens. Note that very strong absolute phase over the whole field of view can be measured; contour effect is due to phase wraps.

Right: (From reference [5]): First demonstration of iterative phase-retrieval electron ptychography. The phase change measurable is factors of $\pi$. The results compare well with a model of the thickness and inner potential of the specimen, FeNi.

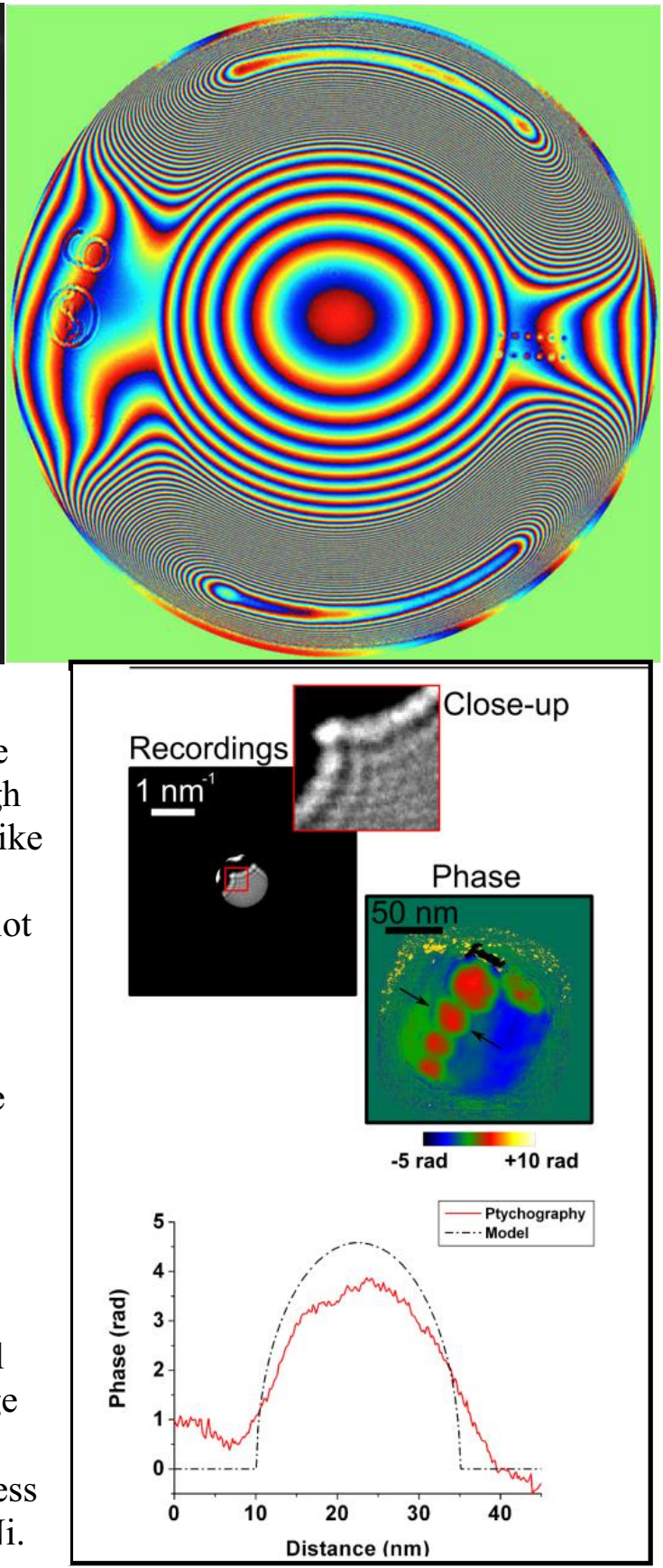

